\title{
Secondhand Smoking and Sudden Infant Death Syndrome: How can in Silico Pharmacokinetics and Circulation Models Contribute?
}

\author{
Harvey $\mathrm{Ho}^{1 *}$, Tingting $\mathrm{Ran}^{2}$ and Xiaojuan $\mathrm{Ji}^{2,3 *}$ \\ ${ }^{1}$ Auckland Bioengineering Institute, The University of Auckland, Auckland, New Zealand, ${ }^{2}$ Children's Hospital of Chongqing \\ Medical University, Chongqing, China, ${ }^{3}$ National Clinical Research Center for Child Health and Disorders, Chongqing Key \\ Laboratory of Pediatrics, Chongqing, China
}

Keywords: SIDS, secondhand smoking, nicotine, pharmacokinetics, computer model

\section{BACKGROUND}

Sudden Infant Death Syndrome (SIDS) is the leading cause of death in infants in developed countries (Moon et al., 2007) (Rogers, 2009). The cardio-respiratory distress due to infant sleep position was considered as the leading risk factor that causes arousal and/or auto-resuscitation deficiency under hypoxia conditions (Moon et al., 2007). The "Back to sleep" campaign of the early 1990s recommended placing sleep babies in a supine position. The campaign led to a substantial decrease in SIDS cases (Rogers, 2009). However, nearly 3 decades later, SIDS remains one of the leading causes of infant death. Among many risk factors, exposure to passive or secondhand smoking (SHS) pre- and postpartum has attracted many research studies (Mitchell et al., 1997) (McMartin et al., 2002) (Albuquerque et al., 2004) (Raghuveer et al., 2016) (Tang et al., 2020). Indeed, SHS exposure and maternal smoking are considered the most modifiable risk factor associated with SIDS (Cnattingius, 2004). It is estimated that if all fetuses had not been exposed to maternal smoking in utero, about one-third of all SIDS deaths might have been prevented (Mitchell et al., 1997).

Blood flow measurements in fetuses and neonates seem to support the viewpoint. The systolic to diastolic (S/D) ratio in the fetal umbilical and middle cerebral arteries is greater in those exposed to maternal smoking than those without exposure (Albuquerque et al., 2004). High S/D ratios are associated with a lower or absent end-diastolic flow, which is typical in fetuses with intrauterine growth restriction (IUGR) (Albuquerque et al., 2004). More direct pieces of evidence are the measurements of nicotine and cotinine (the major metabolite of nicotine) in biopsy tissues of SIDS and non-SIDS victims (McMartin et al., 2002) (Milerad et al., 1994). From the 73 lung samples, McMartin and his co-workers found that there was a significantly higher nicotine concentration in the SIDS cases $(19.64 \pm 2.61 \mathrm{ng} / \mathrm{g})$ than in non-SIDS cases $(7.86 \pm 1.63 \mathrm{ng} / \mathrm{g})$. In contrast, there was no significant difference in cotinine concentrations between SIDS $(10.87 \pm 2.32 \mathrm{ng} / \mathrm{g})$ and non-SIDS cases $(8.71 \pm 1.47 \mathrm{ng} / \mathrm{g})$. In another study (Milerad et al., 1994), it was found that in the pericardial fluid of SIDS victims, about $70 \%$ of the 24 consecutively autopsied cases were moderately (10-20 ng) or heavily (20-50 ng) exposed to SHS.

Early intervention is critical for the prevention of SIDS. However, clinical investigations are hampered by the inaccessibility of fetal, neonatal, and infant tissues and in vivo measurements. Alternatively, animal models, e.g. mice models are used to investigate the effects of SHS (Hu et al., 2012) (Calton et al., 2016). It was found that SHS exposure induced a reduction in the ability of the pre-Bötzinger Complex, a region of inspiratory rhythm generation (Ramirez et al., 2012), to maintain a respiratory rhythm during exposure to severe hypoxia (Hu et al., 2012). However, it is still difficult to verify the underlying mechanisms in animal experiments that involve interweaving factors, as only a limited set of parameters could be tested.

Silico Pharmacokinetics and

Circulation Models Contribute?

Front. Bioeng. Biotechnol. 9:820404.

doi: 10.3389/fbioe.2021.820404 
Another approach, in silico modelling, is valuable because it can test the various hypothesis in a virtual setup. Still, few in silico models are developed for SIDS. A previous article proposed a roadmap for investigating the effects of maternal smoking on cardiovascular risks (Ho et al., 2020). In that roadmap, several components were suggested, including physiologically based pharmacokinetic (PBPK) models, blood flow and endothelial cell signaling models, bioinformatics, stem cell technology, etc. In this Opinion paper, we focus on two kinds of models, i.e. PBPK and circulation models, and their potential applications in SIDS research. We provide a light literature review of existing models, and discuss how they may be customised for the research of SIDS. We also discuss the importance of preparing fundamental data, e.g. longitudinal blood flow to key organs, that are still absent but crucial for developing these models.

\section{PAEDIATRIC PHARMACOKINETIC MODELS}

The purpose of pharmacokinetic models is to quantify the absorption, distribution, metabolism, and excretion (ADME) of a drug/compound in the body. PBPK models use a group of compartments to represent blood, tissues (fat, muscle), and key organs. Differential equations are formulated to model the changes in the amount or concentration of a compound in that compartment (Jones and Rowland-Yeo, 2013). Paediatric PBPK ( $p$-PBPK) models incorporate the physiological and metabolic differences between adults and children, such as the blood flow distribution in individual organs, age-dependent enzyme activities and renal clearance (Amice et al., 2021) (Zhang et al., 2020).

PBPK models of nicotine and cotinine have been recently reported for pregnant women (Amice et al., 2021). However, few in silico studies report the clearance of nicotine and cotinine, the major metabolite of nicotine, in neonates and infants (Gentry et al., 2003). Several factors need to be considered when extrapolating the current models of nicotine metabolism in adults to very young children and fetuses (Moon et al., 2007): neonates and infants have a limited CYP2A6 (the major enzyme that transforms nicotine into cotinine) activity (Jiang et al., 2013); (Rogers, 2009) the volume ratio of brain vs body in fetus/infants is much higher than adults (Mitchell et al., 1997); different circulation routes in fetuses, and the roles played by the placenta and amniotic fluid during pregnancy (Abduljalil et al., 2012). Nicotine can enter the plasma of neonates and infants via inhalation, i.e. through the lung, and also be via breastfeeding through the stomach and intestine (McMartin et al., 2002). P-PBPK models, therefore, need to be adjusted accordingly.

In brief, in silico models are valuable in assessing the absorption and clearance of nicotine in fetuses, neonates, and infants exposed to SHS and the risk of SIDS. Indeed, in silico models may sometimes be the only feasible way. For example, in the case of fetuses, the current clinical practice of sample taking is by analysing blood samples extracted from the umbilical cord but lacking any data from other organs (Huang et al., 2017). In silico models that predict nicotine concentration at early pregnancy or fetal organs like the fetal brain can have a unique value.
It is worth pointing out that parameters are critical in pharmacokinetic models. However, some parameters such as the volume of distribution and the hepatic and renal clearance are often unavailable. Therefore, computational methods, including linear or nonlinear regression, global parameter analysis such as the Latin Hypercube Sampling analysis, are used to fit model results to reported data (Zhang et al., 2020) (Jiang et al., 2013).

\section{CIRCULATION MODELS}

Circulation models are used to investigate the hemodynamics and regulation mechanisms of the cardiovascular system under both healthy and diseased states (Gao et al., 1998). Many computational methods and techniques have been proposed for circulation modelling. They may be roughly grouped according to the spatial dimensions (from $0 \mathrm{D}$ to $3 \mathrm{D}$ ) of the governing equations. For example, 0D models use electronic components such as resistors to represent major arteries and veins, where the temporal blood pressure and flow rate are simulated (Gao et al., 1998). In contrast, $1 \mathrm{D}$ to $3 \mathrm{D}$ models take spatial dimensions of blood vessels (longitudinal and radial dimensions) into the equation, in addition to the time variable (Ho et al., 2009). Models of different dimensions can be combined. For example, a multiscale 0D-3D model was developed where $0 \mathrm{D}$ models were used to represent the systemic, coronary, and pulmonary circulations, whereas the $3 \mathrm{D}$ model was used for the systemic-to-pulmonary shunt (Migliavacca et al., 2006).

The regulation of the cardiovascular system from SIDS is still not well understood. The most relevant vessels to SIDS may be those in the heart, the brain, and the lung. Although many cerebral circulation models have been developed for adults, e.g. in (Gao et al., 1998) (Ho et al., 2009), few cerebral circulation models have been developed for infants. The infant's brain has a similar vascular morphology to adults, though vessel dimensions and electronic component parameters need to be rescaled. In the case of SIDS, the blood supply to the brain stem via the basilar artery can be critical since the regulation centre of respiratory rate exists in the pons, and a gasping centre is thought to be located in the ventrolateral medulla (St.-John et al., 2009).

In silico circulation models could help by testing the hypothesis of poor blood supply, thus hypoxia, associated with SIDS. For example, increased cerebral blood flow velocities were reported in neonates of smoking mothers (Abdul-Khaliq et al., 1993), which can be used as a clue for circulation models. A relevant example is the fetal circulation model of (GarciaCanadilla et al., 2014) that investigates the blood supply to the brains of IUGR subjects, where flow is redistributed to maintain cerebral supply (brain sparing) of different degrees of severity. The hypothesis of poor blood supply to the basilar artery can be simulated by the high resistance of its afferent vessels, the vertebral arteries. This in turn affects the distribution of the cerebral circulation in neonates and infants. Verification of the hypothesis depends upon flow data, which are usually collected from Doppler ultrasonography. 
TABLE 1 | Clinical, experimental or epidemiologic data associated with SHS and SIDS mentioned in the paper

\begin{tabular}{|c|c|c|}
\hline Literature & Data & Reference \\
\hline Milerad et al. (1994) & the pericardial fluid of SIDS victims & Milerad et al. (1994) \\
\hline McMartin et al. (2002) & Lung tissue nicotine/cotinine concentration in SIDS and non-SIDS infants & McMartin et al. (2002) \\
\hline Albuquerque et al. (2004) & Arterial velocity waveform of foetuses exposed to SHS & Albuquerque et al. (2004) \\
\hline Blakely et al. (2004) & Ethnic mortality in infants & Blakely et al. (2004) \\
\hline Hu et al. (2012) & Prenatal nicotine exposure of in vitro respiratory rhythm to hypoxia in mice & Hu et al. (2012) \\
\hline Ramirez et al. (2012) & Activity of the pre-Bötzinger Complex of mice exposed to SHS & Ramirez et al. (2012) \\
\hline Lavezzi (2015) & Pontine Kölliker-Fuse nucleus developments & Lavezzi, (2015) \\
\hline Tang et al. (2020) & SHS survey among pregnant women in a metropolitan city in China & Tang et al. (2020) \\
\hline
\end{tabular}

\section{BLOOD FLOW DATA}

The blood flows in fetuses exposed to maternal smoking were measured by the pulsed Doppler method for large cerebral vessels, as well as umbilical vessels (Albuquerque et al., 2004). Cerebral blood flow velocities were also measured for neonates of smoking mothers (Abdul-Khaliq et al., 1993). In the study of (AbdulKhaliq et al., 1993), it was found that the systolic, mean, and diastolic flow velocity in the anterior, internal carotid, and basilar cerebral arteries of neonates (20-42 h old) were significantly higher than those in the control group, despite their lower birth weight, gestational age, and systolic blood pressure.

However, flow velocities (in $\mathrm{cm} / \mathrm{s}$ ) in blood vessels cannot be translated into flow rates (in $\mathrm{mL} / \mathrm{s}$ ), which is calculated by the product of the flow velocity and the cross-section area. The data for the latter requires measuring the diameter of the artery under the B-mode of ultrasonic imaging. Therefore, both flow velocities and blood vessel diameters are required to verify that the blood supply to the brain stem is an important trigger of SIDS. Unfortunately, literature reporting both flow velocities and vessel diameters in neonates and infant are scant. Even more scarce are the developmental, longitudinal data of the blood flow along with several key time points of infancy. These data are not only important for circulation models, but are also useful for PBPK models because blood flow rates are a key parameter in calculating the distribution of drugs/compounds into different organs.

\section{DISCUSSION}

In this small paper, we have discussed the potential use of in silico circulation and pharmacokinetics models in SIDS research, the modifications that need to be made, and the important data (such as longitudinal blood flow) that are still absent. While new evidence has been found regarding the connection between SHS and SIDS, in silico studies are far lagging behind. For example, abnormal pontine Kölliker-Fuse nucleus (KFN) developments are suspected to be related to SIDS (Lavezzi, 2015). Interestingly, the majority of mothers of infants with altered KFN development were found to be smokers (Lavezzi, 2015). The prevalence of smoking among women exceeds $10 \%$ in many developed countries (Dessì et al., 2018). In the USA, the prevalence of maternal smoking is about 14\% (Dessì et al., 2018). A recent survey found that approximately $30 \%$ of pregnant women were exposed to SHS (more than 15 min per day) in a metropolitan city in China (Tang et al., 2020). In New Zealand, the indigenous Māori infants are six times more likely to die of SIDS than non-Māori New Zealanders (Blakely et al., 2004). Incidentally, Māori women have a much higher pregnancy smoking rate $(32 \%)$ than mothers in other ethnic groups.

This public health concern calls for novel research methodology, including computational models. For cardiovascular models to assess relevant risk factors, important questions need to be asked, such as the cause of fetal hypoxia that impairs brain functions (Pastrakuljic et al., 1998) (Elhaik, 2016), the abnormal respiratory rhythm (Hu et al., 2012), the chronic/ acute effects of nicotine on the cardiovascular system, etc. Before constructing a computer model, thoughts must be put into the physiological/pathological context. For SIDS, in silico pharmacokinetic and circulation models can only provide simulations from their perspectives that need to be placed into the large picture of SIDS. And, while modelling techniques are well established in these disciplines, questions such as which modelling methods are to be chosen, their validation methods are still very challenging. A take-for-granted model may misinterpret the underlying phenomena. In addition, while this paper discusses pharmacokinetic and circulation models only, there are certainly other in silico models, e.g. celluar or molecular kinetics models for hypoxic response could provide fresh ideas underneath SIDS. Numerical methods such as machine learning could assist data analysis such as patient-record of SIDS. Therefore, we believe a closer tie among clinicians, experimentalists and modellers, and a publicly available database of data (Table 1) and in silico models would greatly assist the research of SIDS.

\section{AUTHOR CONTRIBUTIONS}

HH drafted the paper, TR and XJ provided the clinical context of SIDS. All authors reviewed and agreed on the paper.

\section{FUNDING}

$\mathrm{HH}$ acknowledges the partial financial support from the Li Ka Shing Foundation. XJ acknowledges the support from a grant of the Chongqing Municipal Human Resources and Social Security Bureau (cx2019065). 


\section{REFERENCES}

Abdul-Khaliq, H., Segerer, H., Luck, W., and Obladen, M. (1993). Increased Cerebral Blood Flow Velocities in Newborn Infants of Smoking Mothers. Eur. J. Pediatr. 152, 232-235. doi:10.1007/BF01956151

Abduljalil, K., Furness, P., Johnson, T. N., Rostami-Hodjegan, A., and Soltani, H. (2012). Anatomical, Physiological and Metabolic Changes with Gestational Age during Normal Pregnancy. Clin. Pharmacokinet. 51, 365-396. doi:10.2165/ 11597440-000000000-00000

Albuquerque, C. A., Smith, K. R., Johnson, C., Chao, R., and Harding, R. (2004). Influence of Maternal Tobacco Smoking during Pregnancy on Uterine, Umbilical and Fetal Cerebral Artery Blood Flows. Early Hum. Develop. 80, 31-42. doi:10.1016/j.earlhumdev.2004.05.004

Amice, B., Ho, H., Zhang, E., and Bullen, C. (2021). Physiologically Based Pharmacokinetic Modelling for Nicotine and Cotinine Clearance in Pregnant Women. Front. Pharmacol. 12, 688597. doi:10.3389/ fphar.2021.688597

Blakely, T., Ajwani, S., Robson, B., Tobias, M., and Bonné, M. (2004). Decades of Disparity: Widening Ethnic Mortality Gaps from 1980 to 1999. N. Z. Med. J. $117,22$.

Calton, M. A., Howard, J. R., Harper, R. M., Goldowitz, D., and Mittleman, G. (2016). The Cerebellum and SIDS: Disordered Breathing in a Mouse Model of Developmental Cerebellar Purkinje Cell Loss during Recovery from Hypercarbia. Front. Neurol. 7, 78. doi:10.3389/fneur.2016.00078

Cnattingius, S. (2004). The Epidemiology of Smoking during Pregnancy: Smoking Prevalence, Maternal Characteristics, and Pregnancy Outcomes. Nicotine Tob. Res. 6, 125-140. doi:10.1080/14622200410001669187

Dessì, A., Corona, L., Pintus, R., and Fanos, V. (2018). Exposure to Tobacco Smoke and Low Birth Weight: from Epidemiology to Metabolomics. Expert Rev. Proteomics 15, 647-656. doi:10.1080/14789450.2018.1505508

Elhaik, E. (2016). A "Wear and Tear" Hypothesis to Explain Sudden Infant Death Syndrome. Front. Neurol. 7, 180. doi:10.3389/fneur.2016.00180

Gao, E., Young, W. L., Pile-Spellman, J., Ornstein, E., and Ma, Q. (1998). Mathematical Considerations for Modeling Cerebral Blood Flow Autoregulation to Systemic Arterial Pressure. Am. J. Physiology-Heart Circulatory Physiol. 274, H1023-H1031. doi:10.1152/ ajpheart.1998.274.3.h1023

Garcia-Canadilla, P., Rudenick, P. A., Crispi, F., Cruz-Lemini, M., Palau, G., Camara, O., et al. (2014). A Computational Model of the Fetal Circulation to Quantify Blood Redistribution in Intrauterine Growth Restriction. Plos Comput. Biol. 10, e1003667. doi:10.1371/journal.pcbi.1003667

Gentry, P. R., Covington, T. R., and Clewell, H. J. (2003). Evaluation of the Potential Impact of Pharmacokinetic Differences on Tissue Dosimetry in Offspring during Pregnancy and Lactation. Regul. Toxicol. Pharmacol. 38, 1-16. doi:10.1016/S0273-2300(03)00047-3

Ho, H., Sands, G., Schmid, H., Mithraratne, K., Mallinson, G., and Hunter, P. (2009). A Hybrid 1D and 3D Approach to Hemodynamics Modelling for a Patient-specific Cerebral Vasculature and Aneurysm. Med. Image Comput. Computer-Assisted Intervention - MICCAI 12, 323-330. doi:10.1007/978-3642-04271-3_40

Ho, H., Guo, H., Means, S., Tang, J., and Hunter, P. (2020). Maternal Smoking Induced Cardiovascular Risks in Fetuses: How Can In Silico Models Help? Front. Bioeng. Biotechnol. 8, 8. doi:10.3389/fbioe.2020.00097

Hu, H., Brahmbhatt, A., Upadhyaya, R., Vega, D., and Hill, A. A. (2012). Prenatal Nicotine Exposure Alters the Response of the Mouse In Vitro Respiratory Rhythm to Hypoxia. Respir. Physiol. Neurobiol. 181, 234-247. doi:10.1016/ j.resp.2012.03.015

Huang, K.-H., Chou, A.-K., Jeng, S.-F., Ng, S., Hsieh, C.-J., Chen, M.-H., et al. (2017). The Impacts of Cord Blood Cotinine and Glutathione-S-Transferase Gene Polymorphisms on Birth Outcome. Pediatr. Neonatal. 58, 362-369. doi:10.1016/j.pedneo.2016.08.006

Jiang, X.-L., Zhao, P., Barrett, J., Lesko, L., and Schmidt, S. (2013). Application of Physiologically Based Pharmacokinetic Modeling to Predict Acetaminophen Metabolism and Pharmacokinetics in Children. CPT: Pharmacometrics Syst. Pharmacol. 2, 80. doi:10.1038/psp.2013.55
Jones, H., and Rowland-Yeo, K. (2013). Basic Concepts in Physiologically Based Pharmacokinetic Modeling in Drug Discovery and Development. CPT: Pharmacometrics Syst. Pharmacol. 2, 63. doi:10.1038/psp.2013.41

Lavezzi, A. M. (2015). A New Theory to Explain the Underlying Pathogenetic Mechanism of Sudden Infant Death Syndrome. Front. Neurol. 6, 220. doi:10.3389/fneur.2015.00220

McMartin, K. I., Platt, M. S., Hackman, R., Klein, J., Smialek, J. E., Vigorito, R., et al. (2002). Lung Tissue Concentrations of Nicotine in Sudden Infant Death Syndrome (SIDS). J. Pediatr. 140, 205-209. doi:10.1067/mpd.2002.121937

Migliavacca, F., Balossino, R., Pennati, G., Dubini, G., Hsia, T.-Y., de Leval, M. R., et al. (2006). Multiscale Modelling in Biofluidynamics: Application to Reconstructive Paediatric Cardiac Surgery. J. Biomech. 39, 1010-1020. doi:10.1016/j.jbiomech.2005.02.021

Milerad, J., Rajs, J., and Gidlund, E. (1994). Nicotine and Cotinine Levels in Pericardial Fluid in Victims of SIDS. Acta Paediatr. 83, 59-62. doi:10.1111/ j.1651-2227.1994.tb12953.x

Mitchell, E. A., Tuohy, P. G., Brunt, J. M., Thompson, J. M. D., Clements, M. S., Stewart, A. W., et al. (1997). Risk Factors for Sudden Infant Death Syndrome Following the Prevention Campaign in New Zealand: A Prospective Study. Pediatrics 100, 835-840. doi:10.1542/peds.100.5.835

Moon, R. Y., Horne, R. S., and Hauck, F. R. (2007). Sudden Infant Death Syndrome. The Lancet 370, 1578-1587. doi:10.1016/S0140-6736(07)61662-6

Pastrakuljic, A., Schwartz, R., Simone, C., Derewlany, L. O., Knie, B., and Koren, G. (1998). Transplacental Transfer and Biotransformation Studies of Nicotine in the Human Placental Cotyledon Perfused In Vitro. Life Sci. 63, 2333-2342. doi:10.1016/S0024-3205(98)00522-0

Raghuveer, G., White, D. A., Hayman, L. L., Woo, J. G., Villafane, J., Celermajer, D., et al. (2016). Cardiovascular Consequences of Childhood Secondhand Tobacco Smoke Exposure: Prevailing Evidence, Burden, and Racial and Socioeconomic Disparities: A Scientific Statement from the American Heart Association. Circulation 134, e336-e359. doi:10.1161/ CIR.0000000000000443

Ramirez, J. M., Doi, A., Garcia, A. J., Elsen, F. P., Koch, H., and Wei, A. D. (2012). The Cellular Building Blocks of Breathing. Compr. Physiol. 2, 2683-2731. doi:10.1002/cphy.c110033

Rogers, J. M. (2009). Tobacco and Pregnancy. Reprod. Toxicol. 28, 152-160. doi:10.1016/j.reprotox.2009.03.012

St.-John, W. M., Li, A., and Leiter, J. C. (2009). Genesis of Gasping Is Independent of Levels of Serotonin in the Pet-1 Knockout Mouse. J. Appl. Physiol. 107, 679-685. doi:10.1152/japplphysiol.91461.2008

Tang, J., Shen, J., Zhang, S., Ho, H., and Ran, S. (2020). A Pilot Study on Secondhand Smoke Exposure Among Pregnant Women in Chongqing, China: A Combined Questionnaire, Saliva Cotinine Test and Ultrasound Flow index Analysis. Front. Public Health 8, 290. doi:10.3389/ fpubh.2020.00290

Zhang, S., Zhang, E., and Ho, H. (2020). Extrapolation for a Pharmacokinetic Model for Acetaminophen from Adults to Neonates: A Latin Hypercube Sampling Analysis. Drug Metab. Pharmacokinet. 35, 329-333. doi:10.1016/ j.dmpk.2020.03.004

Conflict of Interest: The authors declare that the research was conducted in the absence of any commercial or financial relationships that could be construed as a potential conflict of interest.

Publisher's Note: All claims expressed in this article are solely those of the authors and do not necessarily represent those of their affiliated organizations, or those of the publisher, the editors and the reviewers. Any product that may be evaluated in this article, or claim that may be made by its manufacturer, is not guaranteed or endorsed by the publisher.

Copyright $\odot 2022 \mathrm{Ho}$, Ran and Ji. This is an open-access article distributed under the terms of the Creative Commons Attribution License (CC BY). The use, distribution or reproduction in other forums is permitted, provided the original author(s) and the copyright owner(s) are credited and that the original publication in this journal is cited, in accordance with accepted academic practice. No use, distribution or reproduction is permitted which does not comply with these terms. 